Research Article

\title{
An Extended Newmark-FDTD Method for Complex Dispersive Media
}

\author{
Yu-Qiang Zhang (D) and Peng-Ju Yang (D) \\ School of Physics and Electronic Information, Yanan University, Yan'an 716000, China \\ Correspondence should be addressed to Yu-Qiang Zhang; ya_zyq@aliyun.com
}

Received 18 December 2017; Revised 27 February 2018; Accepted 5 March 2018; Published 10 April 2018

Academic Editor: Luciano Tarricone

Copyright (c) 2018 Yu-Qiang Zhang and Peng-Ju Yang. This is an open access article distributed under the Creative Commons Attribution License, which permits unrestricted use, distribution, and reproduction in any medium, provided the original work is properly cited.

\begin{abstract}
Based on polarizability in the form of a complex quadratic rational function, a novel finite-difference time-domain (FDTD) approach combined with the Newmark algorithm is presented for dealing with a complex dispersive medium. In this paper, the time-stepping equation of the polarization vector is derived by applying simultaneously the Newmark algorithm to the two sides of a second-order time-domain differential equation obtained from the relation between the polarization vector and electric field intensity in the frequency domain by the inverse Fourier transform. Then, its accuracy and stability are discussed from the two aspects of theoretical analysis and numerical computation. It is observed that this method possesses the advantages of high accuracy, high stability, and a wide application scope and can thus be applied to the treatment of many complex dispersion models, including the complex conjugate pole residue model, critical point model, modified Lorentz model, and complex quadratic rational function.
\end{abstract}

\section{Introduction}

The finite-difference time-domain (FDTD) method has been widely used to study electromagnetic (EM) wave interaction with a wide variety of materials due to its robustness and its ability to calculate accurate broadband response via a single simulation $[1,2]$. In the analysis of dispersive materials using the FDTD approach, it is required that the variation of dielectric parameters with frequency is modeled efficiently and accurately. In recent years, some novel dispersive models have been introduced, for example, the complex-conjugate pole-residue (CCPR) model [3], critical point (CP) model [4], modified Lorentz (m-Lo) model [5], and quadratic complex rational function (QCRF) model [6]. Some hybrid dispersion models, such as the DrudeLorentz model $[7,8]$ and Drude-CP model $[4,9]$, were also usually adopted. However, the introduction of these new dispersion models and their hybrid models undoubtedly challenges the versatility of the existing FDTD methods. For the typical dispersive FDTD approaches, the recursive convolution (RC) method and $Z$ transform (ZT) method are not convenient to deal with the high-order models such as $\mathrm{m}$-Lo and QCRF. The auxiliary differential equation (ADE) method has more stringent stability conditions than the Courant-Friedrichs-Lewy (CFL) condition of Yee's FDTD scheme [10-12].

The Newmark algorithm was originally used for the numerical solution of structural dynamics and was introduced into the finite-element time-domain (FETD) method for electromagnetic simulations [13-15]. Then, it is introduced into the analysis of the dispersive FDTD computation in 2014 for the unified treatment of the Debye, Drude, and Lorentz medium [16-18]. In this paper, the NewmarkFDTD method is further extended to the high-order model so that it can be used as a general approach for dispersive media, not only to treat with Debye, Drude, and Lorentz media, but also to deal with complex dispersion models including CCPR, CP, m-Lo, and QCRF media. The scope of application of the algorithm is extended greatly. Then, the computational accuracy and stability are investigated from the two aspects of theory and numerical computation, and higher accuracy and stability are shown. 


\section{Extended Newmark-FDTD Method}

For the linear electric dispersive medium, the Maxwell's curl equation can be written as

$$
\begin{aligned}
& \nabla \times \mathbf{H}=\frac{\partial \mathbf{D}}{\partial t}+\sigma \mathbf{E}, \\
& \nabla \times \mathbf{E}=-\frac{\partial \mathbf{B}}{\partial t},
\end{aligned}
$$

where E, D, H, and $\mathbf{B}$ are the electric field strength, electric displacement, magnetic field strength, and magnetic flux density, respectively. $\sigma$ is the electric conductivity. For simplicity, consider only the electric dispersion medium. The constitutive relation in a frequency domain can be characterized as

$$
\begin{aligned}
\mathbf{B} & =\mu \mathbf{H}, \\
\mathbf{D}(\omega) & =\varepsilon(\omega) \mathbf{E}(\omega)=\varepsilon_{0}\left[\varepsilon_{\infty}+\chi(\omega)\right] \mathbf{E}(\omega),
\end{aligned}
$$

where $\mu$ is the magnetic permeability. $\varepsilon_{0}$ and $\varepsilon_{\infty}$ are the relative electric permittivity in free space and at the infinite frequency, respectively. $\varepsilon(\omega)$ and $\chi(\omega)$ are the electric permittivity and electric susceptibility in frequency domain, respectively. In this paper, the electric susceptibility is adopted in the following rational function form:

$$
\chi(\omega)=\sum_{q=1}^{Q} \chi_{q}(\omega)=\sum_{q=1}^{Q} \frac{b_{0, q}+b_{1, q}(j \omega)+b_{2, q}(j \omega)^{2}}{a_{0, q}+a_{1, q}(j \omega)+a_{2, q}(j \omega)^{2}}
$$

where $a_{i, q}$ and $b_{i, q}(i=0,1,2)$ are both real constants. In this way, the three typical kinds of dispersive models, that is, Debye, Drude, and Lorentz, and some of the complex dispersive models, that is, CCPR, CP, m-Lo, and QCRF, can be viewed as special cases of (5). The higher degrees of freedom and wider range of application are possessed.

The time-stepping equation of $\mathbf{H}$ can be simply obtained by applying the Yee discrete scheme to (2). However, to obtain the time-stepping equation of $\mathbf{E}$ from (1), we must firstly solve (4) in a time-stepping manner. For this reason, the polarization vector $\mathbf{P}$ in the frequency domain is introduced

$$
\mathbf{P}(\omega)=\chi(\omega) \mathbf{E}(\omega)
$$

and let $\mathbf{P}_{q}=\chi_{q}(\omega) \mathbf{E}(\omega)$; thus, (4) can further be represented as

$$
\mathbf{D}(\omega)=\varepsilon_{0} \varepsilon_{\infty} \mathbf{E}(\omega)+\varepsilon_{0} \sum_{q=1}^{Q} \mathbf{P}_{q}(\omega)
$$

Substituting (5) into $\mathbf{P}$, we can obtain

$$
\begin{aligned}
& {\left[a_{0, q}+a_{1, q}(j \omega)+a_{2, q}(j \omega)^{2}\right] \mathbf{P}_{q}(\omega)} \\
& =\left[b_{0, q}+b_{1, q}(j \omega)+b_{2, q}(j \omega)^{2}\right] \mathbf{E}(\omega) .
\end{aligned}
$$

Converting (8) into a time domain by the relation $j \omega \rightarrow \partial / \partial t$, we arrive at a two-order differential equation

$$
\begin{aligned}
a_{2, q} \frac{d^{2} \mathbf{P}_{q}(t)}{d t^{2}}+a_{1, q} & \frac{d \mathbf{P}_{q}(t)}{d t}+a_{0, q} \mathbf{P}_{q}(t) \\
& =\left[b_{2, q} \frac{d^{2} \mathbf{E}(t)}{d t^{2}}+b_{1, q} \frac{d \mathbf{E}(t)}{d t}+b_{0, q}\right] \mathbf{E}(t) .
\end{aligned}
$$

It can be observed that the right side of (9) not only contains $\mathbf{E}(t)$ but also contains the first-order derivative and secondorder derivative of $\mathbf{E}(t)$ so that it cannot be solved directly using the Newmark algorithm employed in [16, 17]. For this reason, a temporary variable $\mathbf{W}$ is introduced and (9) is further represented as

$$
\begin{gathered}
a_{2, q} \frac{d^{2} \mathbf{P}_{q}(t)}{d t^{2}}+a_{1, q} \frac{d \mathbf{P}_{q}(t)}{d t}+a_{0, q} \mathbf{P}_{q}(t)=\mathbf{W}, \\
b_{2, q} \frac{d^{2} \mathbf{E}(t)}{d t^{2}}+b_{1, q} \frac{d \mathbf{E}(t)}{d t}+b_{0, q} \mathbf{E}(t)=\mathbf{W} .
\end{gathered}
$$

Then, the Newmark algorithm is applied to (10) with a unified time step $\Delta t$ and to obtain higher stability, $\gamma=0.5$ and $\beta=0.25$ are adopted $[13,14]$. Thus, we can obtain

$$
\begin{aligned}
c_{0, q} \cdot \mathbf{P}_{q}^{n+1}-c_{1, q} & \cdot \mathbf{P}_{q}^{n}+c_{2, q} \cdot \mathbf{P}_{q}^{n-1} \\
& =\frac{(\Delta t)^{2}}{4} \mathbf{W}^{n+1}+\frac{(\Delta t)^{2}}{2} \mathbf{W}^{n}+\frac{(\Delta t)^{2}}{4} \mathbf{W}^{n-1} \\
d_{0, q} \cdot \mathbf{E}^{n+1}-d_{1, q} \cdot & \mathbf{E}^{n}+d_{2, q} \cdot \mathbf{E}^{n-1} \\
& =\frac{(\Delta t)^{2}}{4} \mathbf{W}^{n+1}+\frac{(\Delta t)^{2}}{2} \mathbf{W}^{n}+\frac{(\Delta t)^{2}}{4} \mathbf{W}^{n-1},
\end{aligned}
$$

where

$$
\begin{aligned}
& c_{0, q}=a_{2, q}+\frac{1}{2} a_{1, q} \Delta t+\frac{1}{4} a_{0, q}(\Delta t)^{2}, \\
& c_{1, q}=2 a_{2, q}-\frac{1}{2} a_{0, q}(\Delta t)^{2}, \\
& c_{2, q}=a_{2, q}-\frac{1}{2} a_{1, q} \Delta t+\frac{1}{4} a_{0, q}(\Delta t)^{2} .
\end{aligned}
$$

$d_{0, q}, d_{1, q}$, and $d_{2, q}$ are similar to $c_{0, q}, c_{1, q}$, and $c_{2, q}$. It only needs replacing $a_{0, q}, a_{1, q}$, and $a_{2, q}$ in (13), (14), and (15) with $b_{0, q}$, $b_{1, q}$, and $b_{2, q}$.

Due to be a unified $\Delta t, \mathbf{W}$ in (11) and (12), the value of $\mathbf{W}$ at $n+1, n$, and $n-1$ must be the same, so we can obtain the $\mathbf{P}_{q}$ update formula

$$
\mathbf{P}_{q}^{n+1}=\frac{1}{c_{0, q}}\left[c_{1, q} \cdot \mathbf{P}_{q}^{n}-c_{2, q} \cdot \mathbf{P}_{q}^{n-1}+d_{0, q} \cdot \mathbf{E}^{n+1}-d_{1, q} \cdot \mathbf{E}^{n}+d_{2, q} \cdot \mathbf{E}^{n-1}\right] .
$$


Finally, substituting (16) and (7) into the discretized form of (1), the FDTD-updating formula of $\mathbf{E}$ is given

$$
\begin{aligned}
\left(\varepsilon_{0} \varepsilon_{\infty}+\varepsilon_{0} \sum_{q=1}^{Q} \frac{d_{0, q}}{c_{0, q}}+\frac{\sigma \Delta t}{2}\right) \mathbf{E}^{n+1} \\
=\left(\varepsilon_{0} \varepsilon_{\infty}+\varepsilon_{0} \sum_{q=1}^{Q} \frac{d_{1, q}}{c_{0, q}}-\frac{\sigma \Delta t}{2}\right) \mathbf{E}^{n}+\Delta t \cdot \nabla \times \mathbf{H} \\
\\
-\left(\varepsilon_{0} \sum_{q=1}^{Q} \frac{d_{2, q}}{c_{0, q}}\right) \mathbf{E}^{n-1} \\
+\varepsilon_{0} \cdot\left[\sum_{q=1}^{Q} \frac{\left(1-c_{1, q}\right)}{c_{0, q}} \cdot \mathbf{P}_{q}^{n}+\sum_{q=1}^{Q} \frac{c_{2, q}}{c_{0, q}} \cdot \mathbf{P}_{q}^{n-1}\right]
\end{aligned}
$$

In this way, a complete FDTD time-domain iterative computation is constituted by (17) and (16) and the standard FDTD formula of $\mathbf{H}$ corresponding to (2). The FDTD computation can be realized.

\section{Analysis of Accuracy and Stability}

In the FDTD method, Maxwell's curl equations are replaced with a different equation and the dispersion relation in the discrete form is thus quite different from that in the continuous form. For the dispersive FDTD method, the accuracy of the FDTD method is related also to a discrete scheme applied to the dispersive model of the media in the time domain. So, the accuracy of FDTD method can clearly be understood by investigating the errors between the numerical dispersive model of media and analytical model of media. The smaller the errors, the higher the computational accuracy [19]. The accuracy of the extended NewmarkFDTD algorithm is discussed below. Moreover, for simplicity and without loss of generality, let $Q=1$ and $\varepsilon_{\infty}=0$; the permittivity of dispersive media becomes a rational fraction form similar to (5).

To investigate the accuracy of the extended FDTD method, the $z$-domain electric susceptibility is given by applying the $z$ transform to (16)

$$
\tilde{\chi}(z)=\frac{d_{0, q} z-d_{1, q}+d_{2, q} z^{-1}}{c_{0, q} z-c_{1, q}+c_{2, q} z^{-1}}
$$

Substituting $c_{0, q}, c_{1, q}, c_{2, q}, d_{0, q}, d_{1, q}, d_{2, q}$, and $z=e^{j \omega \Delta t}$ into (18) and then rearranging the resulting equation, we can arrive at

$$
\tilde{\chi}\left(e^{j \omega}\right)=\frac{b_{0, q}(\Delta t)^{2} \cos ^{2}((1 / 2) \omega \Delta t)+j b_{1, q}(\Delta t) \sin (\omega \Delta t)+4 b_{2, q} \sin ^{2}((1 / 2) \omega \Delta t)}{a_{0, q}(\Delta t)^{2} \cos ^{2}((1 / 2) \omega \Delta t)+j a_{1, q}(\Delta t) \sin (\omega \Delta t)+4 a_{2, q} \sin ^{2}((1 / 2) \omega \Delta t)} .
$$

To derive a more compact form, the following double-angle formula of trigonometric identity is applied to (19):

$$
\begin{aligned}
\sin (\omega \Delta t) & =2 \sin \left(\frac{1}{2} \omega \Delta t\right) \cos \left(\frac{1}{2} \omega \Delta t\right), \\
\tan \left(\frac{1}{2} \omega \Delta t\right) & =\frac{\sin \left(\frac{1}{2} \omega \Delta t\right)}{\cos \left(\frac{1}{2} \omega \Delta t\right)} .
\end{aligned}
$$

The numerical electric susceptibility corresponding to (5) is derived as follows:

$$
\tilde{\chi}(\tilde{\omega})=\frac{b_{0}+b_{1}(j \tilde{\omega})+b_{2}(j \tilde{\omega})^{2}}{a_{0}+a_{1}(j \tilde{\omega})+a_{2}(j \tilde{\omega})^{2}}
$$

where

$$
\tilde{\omega}=\frac{2}{\Delta t} \tan \left(\frac{1}{2} \omega \Delta t\right)
$$

Comparing (21) with (5), it is shown that the forms of numerical electric susceptibility $\tilde{\chi}(\tilde{\omega})$ and electric susceptibility $\chi(\omega)$ are exactly the same, apart from replacing $\omega$ with $\tilde{\omega}$.

By expanding (22) into the power series, we can obtain

$$
2 \tan \left(\frac{\omega \Delta t}{2}\right)=\omega \Delta t+\frac{(\omega \Delta t)^{3}}{12}+O(\omega \Delta t)^{5} \approx \omega \Delta t
$$

The error between $\tilde{\omega}$ and $\omega$ is not higher than $(\omega \Delta t)^{3}$. For practical FDTD computation, $\omega \Delta t$ restricted by CFL stable condition is always very small. Hence, $\tilde{\chi}(\tilde{\omega})$ is very close to $\chi(\omega)$. In comparison with the $\mathrm{ADE}$ method given in $[10,19]$, the extended Newmark-FDTD method possesses higher accuracy.

Then, the stability of the extended FDTD method is investigated, which is of great importance to fully employ this method for general dispersive media. The stability of the dispersive FDTD method depends not only on the dispersive model but also on the discretization scheme [11]. It can be carried out by the Von Neumann method combined with the Hurwitz-Routh (R-H) criterion [20]. Consider a timedependent wave equation in a source-free homogeneous dispersive medium, we have

$$
(z-1)^{2} \tilde{\mathbf{D}}+4 z \varepsilon_{0} v^{2} \tilde{\mathbf{E}}=0
$$


where

$$
v^{2}=\left(c_{0} \Delta t\right)^{2} \sum_{s=x, y, z} \sin ^{2} \frac{\left(\tilde{k}_{s} \Delta s / 2\right)}{(\Delta s)^{2}}
$$

In (24) and (25), $\tilde{\mathbf{D}}$ and $\tilde{\mathbf{E}}$ are complex amplitudes of field quantities, $\tilde{k}_{s}(s=x, y, z)$ is a numerical wavenumber in $s$ direction, $\Delta s$ is the space step in $s$ direction, and $c_{\mathrm{\infty}}=1 / \sqrt{\mu_{0} \varepsilon_{\mathrm{\infty}}}$. Substituting (18) into (4), we have

$$
\tilde{\varepsilon}(z)=\frac{\tilde{\mathbf{D}}}{\tilde{\mathbf{E}}}=\varepsilon_{0} \frac{d_{0} z^{2}-d_{1} z+d_{2}}{c_{0} z^{2}-c_{1} z+c_{2}} .
$$

The stability polynomial in $z$-plane can be given as follows from (24) and (26):

$$
\begin{aligned}
S_{D E}(z)= & d_{0} z^{4}-\left[d_{1}+2 d_{0}-4 c_{0} v^{2}\right] z^{3} \\
& +\left[d_{2}+2 d_{1}+d_{0}-4 c_{1} v^{2}\right] z^{2} \\
& -\left[2 d_{2}+d_{1}-4 c_{2} v^{2}\right] z+d_{2}
\end{aligned}
$$

It is known from the knowledge of signal processing that the root of $S_{D E}(z)$ must lie in the unit circle on the $z$-plane to make the FDTD algorithm stable. In order to avoid solving directly the root of $S_{D E}(z)$, we can transform (27) into the $s$-plane by the relation $z=(s+1) /(s-1)$, then substituting $c_{0}, c_{1}, c_{2}, d_{0}, d_{1}$, and $d_{2}$, the following stability polynomial in the $s$-plane is obtained:

$$
\begin{aligned}
S_{D E}(s)= & 4 v^{2} a_{0}(\Delta t)^{2} s^{4}+8 v^{2} a_{1}(\Delta t) s^{3} \\
& +\left[4\left(b_{0}-v^{2} a_{0}\right)(\Delta t)^{2}+16 v^{2} a_{2}\right] s^{2} \\
& +\left[8\left(b_{1}-v^{2} a_{1}\right)(\Delta t)\right] s+16\left(b_{2}-v^{2} a_{2}\right) .
\end{aligned}
$$

In this way, the problem of judgment of whether the root of $S_{D E}(z)$ is in the unit circle on the $z$-plane is converted into the problem of whether the root of $S_{D E}(s)$ lies in the left of the $s$-plane, which can be calculated according to the R-H criterion with the Routh table. Finally, the following effective stability conditions are obtained:

$$
\begin{aligned}
a_{0} & \geq 0, \\
a_{1} & \geq 0, \\
\left(a_{1} b_{0}-a_{0} b_{1}\right)(\Delta t)^{2}+4 v^{2} a_{1} a_{2} & \geq 0, \\
b_{2}-a_{2} \varepsilon_{0} v^{2} & \geq 0 .
\end{aligned}
$$

Taking (32) into account, for $a_{2}>0$, the last numerical stability condition of the extended Newmark-FDTD method can be written in terms of $\Delta t$ :

$$
\Delta t \leq \sqrt{\frac{b_{2}}{a_{2}}} \frac{1}{c_{0}}\left(\sum_{s=x, y, z} \frac{1}{\Delta s^{2}}\right)^{-1 / 2} .
$$

A comparison of the extended Newmark-FDTD algorithm with the double average scheme (DAS) of the ADE method as well as the Mobius transformation (MT) method indicates that a similar time-domain update formula is presented

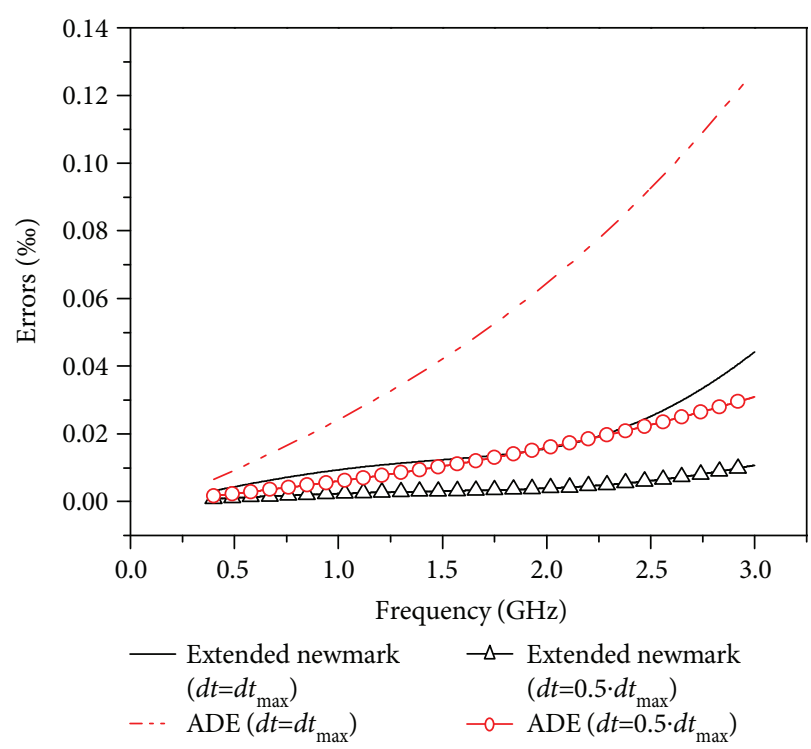

FIgURE 1: The relative error of $\tilde{\varepsilon}(\tilde{\omega})$ and $\varepsilon(\omega)$ for the extended Newmark algorithm.

except for the coefficient difference with a constant in the three dispersive FDTD algorithms, although these three formulations are based on different approximation principles $[11,21-23]$. The aforementioned numerical stability condition expressed by (33) is also the same as the stability condition of DAS and MT and is more relaxed than the stability condition of the direct scheme (DS) of the ADE algorithm [12]. Therefore, the extended Newmark-FDTD method also has the best stability $[24,25]$. However, the derivation of the extended Newmark scheme is much simpler than that of the ADE and MT method, as the problem of the discrete scheme and time synchronism in the ADE algorithm as well as the tedious derivation in the MT algorithm can be avoided $[22,26]$.

\section{Numerical Verification}

To verify the advantages of extended Newmark-FDTD method, the numerical analysis of accuracy and stability of this method is further conducted.

Firstly, to verify the computational accuracy of the extended Newmark-FDTD algorithm, consider a 1-D human dry skin tissue modeled in the frequency range of $400 \mathrm{MHz}-$ $3 \mathrm{GHz}$ by a QCRF with the coefficients of $Q=1, b_{0}=188.85$, $b_{1}=7.49 \times 10^{-8}, b_{2}=1.04 \times 10^{-18}, a_{0}=1.0, a_{1}=1.80 \times 10^{-9}$, and $a_{2}=4.38 \times 10^{-20}$ [19]. The error is measured using the relative error between $\tilde{\varepsilon}(\tilde{\omega})$ and $\varepsilon(\omega)$ defined as

$$
\text { error }=\frac{|\varepsilon(\omega)-\tilde{\varepsilon}(\tilde{\omega})|}{|\varepsilon(\omega)|} .
$$

The results at $d t=d t_{\max }$ and $d t=0.5 \cdot d t_{\max }$ are shown in Figure 1 , where $d t_{\max }=\Delta z / c_{0}$ and $\Delta z$ are one tenths of the minimum wavelength in incident wave. As a comparison, the relative error of the direct scheme (DS) of the ADE method is also given. It is seen from Figure 1 that both the 


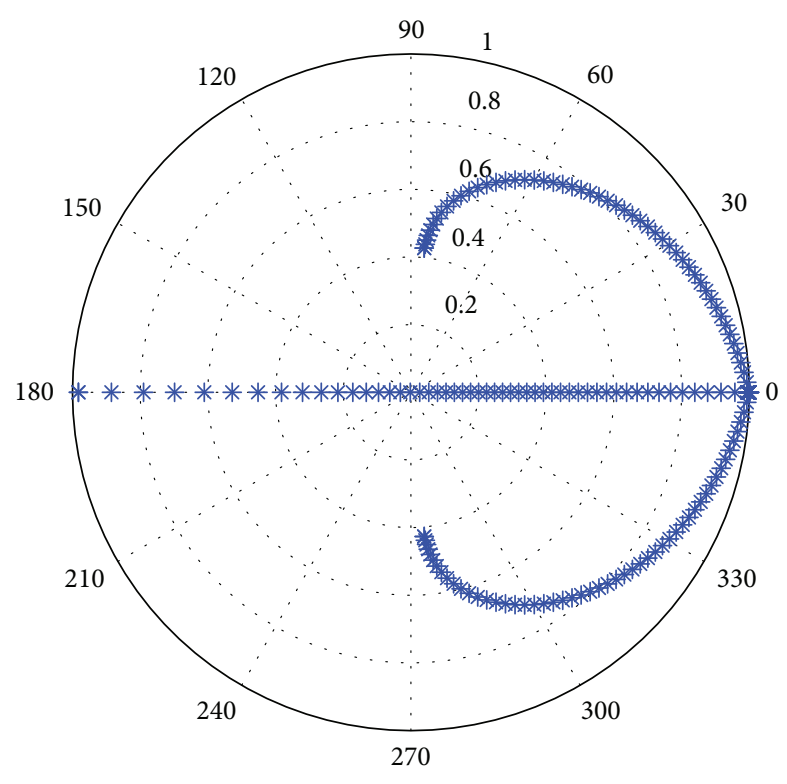

(a) Extended Newmark-FDTD method

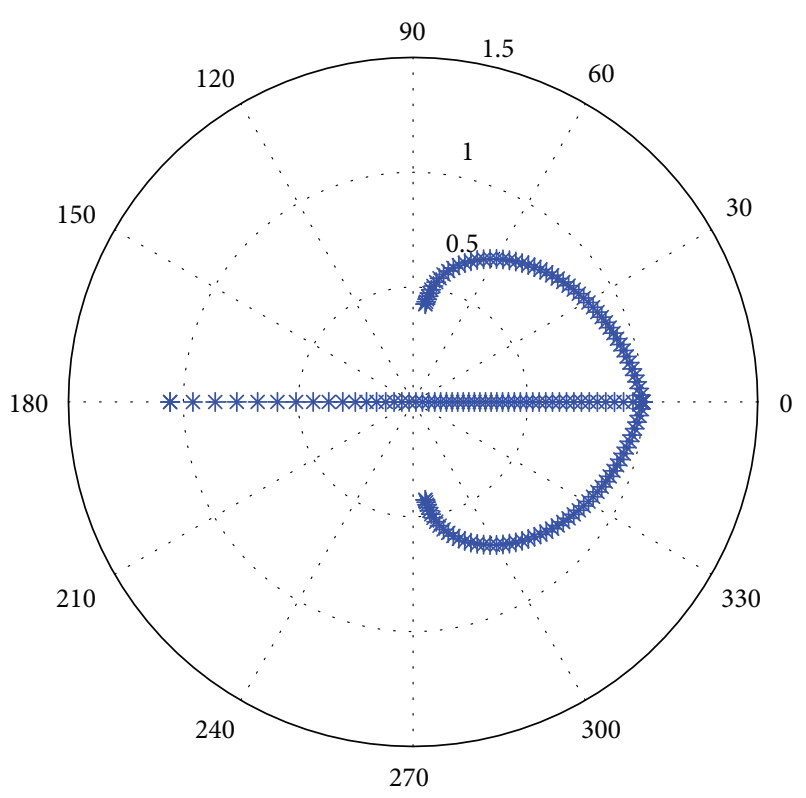

(b) Direct scheme of ADE method

FIgURE 2: Root locus of the stability polynomial in $z$-domain.

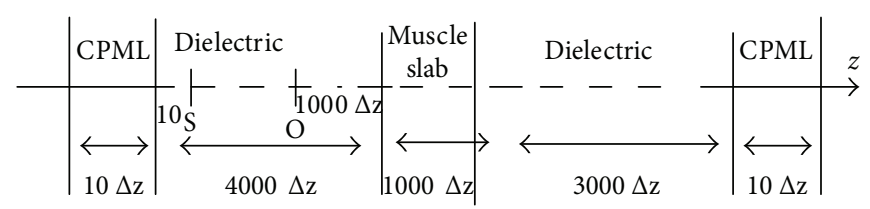

FIGURE 3: Structure of simulation domain (where $\mathrm{S}$ and $\mathrm{O}$ are, resp., the source point and observation point).

relative errors of the extended Newmark algorithm and ADE algorithm are very small and decrease with the decrease of the discrete interval $\Delta t$. A comparison of the two methods shows that the extended Newmark algorithm has a smaller magnitude of relative error than the ADE method, so it has higher computational accuracy.

The stability of the extended Newmark-FDTD method is also investigated by the root locus of the stability polynomial in $z$-plane as (27). Consider a human muscle tissue modeled in the frequency range of $400 \mathrm{MHz}-3 \mathrm{GHz}$ by QCRF as (5) with the following parameters [21]: $Q=1, \varepsilon_{\infty}=0$, $b_{0}=455.72, b_{1}=2.50 \times 10^{-7}, b_{2}=1.98 \times 10^{-18}, a_{0}=1, a_{1}=$ $4.47 \times 10^{-9}$, and $a_{2}=7.56 \times 10^{-20}$. Let $\lambda_{\min }=0.0138 \mathrm{~m}$ and $\Delta z=\lambda_{\min } / 10$, then the maximum time step satisfying the CFL stability limit of 1-D FDTD in free space is $\Delta t_{0}=$ $\Delta z / c_{0}=4.6021 \mathrm{ps}$ and the time step of 1-D FDTD in human muscle tissue is $\Delta t=v \cdot \Delta t_{0}$, where $0<v \leq v_{\max }=\sqrt{b_{2} / a_{2}}=$ 5.1177, $v_{\max }$ is computed from (33). Then, the root locus of the stability polynomial of an extended Newmark-FDTD implementation is computed and shown in Figure 2(a) for $0<v \leq v_{\max }$. At the same time, the root locus of the stability polynomial of the direct scheme of the ADE algorithm is given in Figure 2(b).

It is seen from Figure 2(a) that for $0<\mathrm{v}<5.1177$, all the roots of the stability polynomial of the extended Newmark-FDTD implementation are inside the unit circle that shows the extended Newmark-FDTD method is stable for $\Delta t$ satisfied (33). This is consistent with the theoretical analysis. On the other hand, it is seen from Figure 2(b) that one root of the stability polynomial of the DS-ADE method escapes outside the unit circle for $5.0422<v<5.1177$. This indicates that the direct scheme of the ADE method becomes unstable. This result is also consistent with the results from [21]. The comparison of Figures 2(a) and 2(b) shows that the stability condition of the extended Newmark scheme is more relaxed than that of the ADE direct implementation scheme.

To validate further the theoretical stability results, the FDTD simulation of electromagnetic wave propagation in a 1-D domain is considered. The simulation domain is composed of a human muscle tissue slab with the same parameters as described above and sandwiched between two semi-infinite dielectrics with $\varepsilon_{r}=A_{2} / B_{2}+1$. The size of the simulation domain is limited to 8000 space cells and terminated by the convolutional perfectly matched layer (CPML) with a thickness of 10 cells. The muscle slab is located between the nodes 4000 and 5000 of the domain. A modulated Gaussian pulse given by $E_{x}=\exp \left(-(t / \tau-3)^{2}\right) \sin$ $\left(2 \pi f_{c} t\right)$ is introduced at the $S$ point that is $10 \Delta z$ away from the left boundary of the simulation domain (where $f_{c}=1.5 \mathrm{GHz}$ and $\tau=0.5198 \mathrm{~ns}$ ). The electric field $E_{x}$ can be observed at point $\mathrm{O}$ that is $3000 \Delta z$ away from the left boundary of the simulation domain [21]. The simulation domain geometry is shown in Figure 3. 


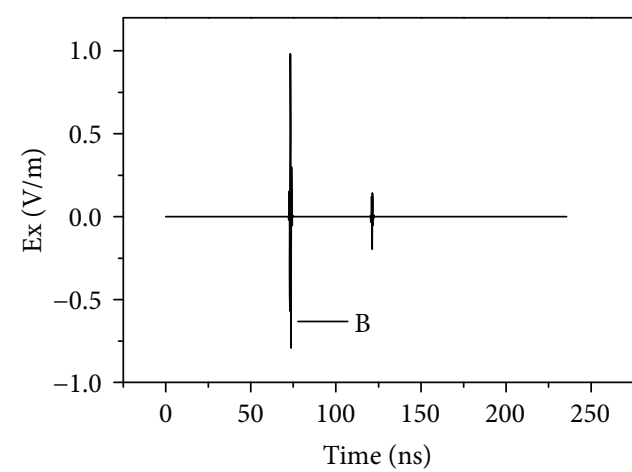

(a) $\Delta t=\Delta t_{\max }$

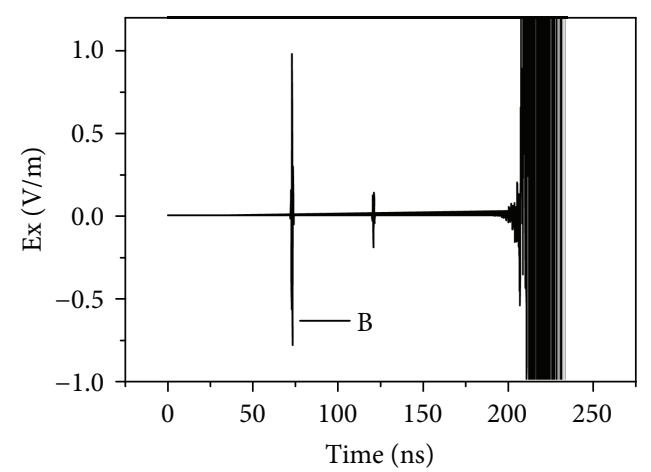

(b) $\Delta t=1.01 \Delta t_{\max }$

Figure 4: Waveform of $E_{x}$ at the observation point.

If the spatial step is $\Delta z=0.00138 \mathrm{~m}$, the maximum time step of the 1-D FDTD computation in free space that satisfied the Courant stability condition is $\Delta t_{0}=\Delta z / c_{0}=$ $4.6021 \mathrm{ps}$ and the upper bound of the time step of $1-\mathrm{D}$ FDTD in the human muscle tissue for the discretization of extended Newmark FDTD is $\Delta t_{\max }=\sqrt{A_{2} / B_{2}} \Delta t_{0}=$ $5.1177 \Delta t_{0}=23.5521 \mathrm{ps}$ obtained from (33). The maximum time step of the 1-D FDTD computation in the surrounding semi-infinite dielectrics is $\Delta t=\Delta z /\left(c_{0} / \sqrt{\varepsilon_{r}}\right)=23.997 \mathrm{ps}$. In the first test, the FDTD simulation is performed with a time step limit $\Delta t_{\max }$. This time step selection meets the stability limit in the human muscle tissue. Figure 4(a) shows the time-domain evolution of $E_{x}$ as recorded at the observation point $O$. It can be seen that the field $E_{x}$ remains stable during the whole period of time. The simulation is repeated by using $\Delta t=1.01 \Delta t_{\max }=23.7877 \mathrm{ps}$ that is bigger than the stability limit in the human muscle tissue. Figure 4(b) shows the time-domain evolution of $E_{x}$ as recorded at observation point $\mathrm{O}$. It can be seen that the field begins to increase without being bound in the late-time response, and therefore, it is unstable.

\section{Conclusion}

In this paper, an extended Newmark-FDTD approach for the complex dispersive medium is presented in terms of polarizability in the form of a quadratic complex rational function, in which the time-stepping equation of the polarization vector is obtained by the Newmark algorithm used widely in finite-element time-domain (FETD) computation. The high accuracy and stability of this method are demonstrated from the two aspects of theoretical analyses and numerical results. It is also indicated that the application scope of this approach is extended greatly. In addition, this approach can be further used for the analysis of some complex dispersive models and some hybrid models, for example, CCPR, CP, m-Lo, QCRF, Drude-Lorentz, and Drude-CP.

\section{Conflicts of Interest}

The authors declare that they have no conflicts of interest.

\section{Acknowledgments}

This project is supported by the National Natural Scientific Foundation of China (Grant no. 61701428) and the Foundation of Construction of High-Level University Project in Shaanxi Province, China (Grant no. 2015SXTS02).

\section{References}

[1] K. Yee, "Numerical solution of initial boundary value problems involving Maxwell's equations in isotropic media," IEEE Transactions on Antennas and Propagation, vol. 14, no. 3, pp. 302-307, 1966.

[2] A. Taflove and S. C. Hagness, Computational Electromagnetics: Finite-Difference Time-Domain Method, Artech House, Norwood, MA, USA, 3rd edition, 2005.

[3] M. Han, R. W. Dutton, and S. Fan, "Model dispersive media in finite-difference time-domain method with complex-conjugate pole-residue pairs," IEEE Microwave and Wireless Components Letters, vol. 16, no. 3, pp. 119-121, 2006.

[4] K. P. Prokopidis and D. C. Zografopoulos, "Efficient FDTD algorithms for dispersive Drude-critical points media based on bilinear z-transform," Electronics Letters, vol. 49, no. 8, pp. 534-536, 2013.

[5] K. P. Prokopidis and D. C. Zografopoulos, "Investigation of the stability of ADE-FDTD methods for modified Lorentz media," IEEE Microwave and Wireless Components Letters, vol. 24, no. 10, pp. 659-661, 2014.

[6] S. G. Ha, J. Cho, J. Choi, H. Kim, and K. Y. Jung, "FDTD dispersive modeling of human tissues based on quadratic complex rational function," IEEE Transactions on Antennas and Propagation, vol. 61, no. 2, pp. 996-999, 2013.

[7] A. Vial, A. S. Grimault, D. Macias, D. Barchiesi, and M. L. de la Chapelle, "Improved analytical fit of gold dispersion: application to the modeling of extinction spectra with a finite-difference time-domain method," Physical Review B, vol. 71, no. 8, 2005.

[8] W. H. P. Pernice, F. P. Payne, and D. F. G. Gallagher, "A general framework for the finite-difference time-domain simulation of real metals," IEEE Transactions on Antennas and Propagation, vol. 55, no. 3, pp. 916-923, 2007.

[9] A. Vial and T. Laroche, "Description of dispersion properties of metals by means of the critical points model and application to the study of resonant structures using the 
FDTD method," Journal of Physics D: Applied Physics, vol. 40, no. 22, pp. 7152-7158, 2007.

[10] S. M. Park, E. K. Kim, Y. B. Park, S. Ju, and K. Y. Jung, "Parallel dispersive FDTD method based on the quadratic complex rational function," IEEE Antennas and Wireless Propagation Letters, vol. 15, pp. 425-428, 2016.

[11] J. Cho, S. G. Ha, Y. B. Park, H. Kim, and K. Y. Jung, "On the numerical stability of finite-difference time-domain for wave propagation in dispersive media using quadratic complex rational function," Electromagnetics, vol. 34 , no. 8, pp. 625632, 2014.

[12] O. Ramadan, "Stability considerations for the direct FDTD implementation of the dispersive quadratic complex rational function models," IEEE Transactions on Antennas and Propagation, vol. 64, no. 11, pp. 4929-4932, 2016.

[13] N. M. Newmark, "A method of computation for structural dynamics," Journal of Engineering Mechanics Division, vol. 85, pp. 67-94, 1959.

[14] O. C. Zienkiewicz, "A new look at the Newmark, Houbolt and other time stepping formulas. A weighted residual approach," Earthquake Engineering \& Structural Dynamics, vol. 5, no. 4, pp. 413-418, 1977.

[15] D. Jiao and J.-M. Jin, "A general approach for the stability analysis of the time-domain finite-element method for electromagnetic simulations," IEEE Transactions on Antennas and Propagation, vol. 50, no. 11, pp. 1624-1632, 2002.

[16] B. Wei, L. Cao, F. Wang, and Q. Yang, "Frequency-dependent FDTD algorithm using Newmark's method," International Journal of Antennas and Propagation, vol. 2014, Article ID 216763, 6 pages, 2014.

[17] F. Wang, B. Wei, and L. Q. Li, "Newmark method for finitedifference time-domain modeling of wave propagation in frequency-dispersive medium," Acta Physica Sinica, vol. 63, no. 13, 2014.

[18] F. Wang, B. Wei, Q. Yang, and L. Li, "FDTD formulation for dispersive chiral media using the Newmark algorithm," Journal of Xidian University, vol. 44, no. 1, pp. 44-50, 2017.

[19] K. Y. Jung, "On the numerical accuracy of finite-difference time-domain dispersive modeling based on a complex quadratic rational function," Electromagnetics, vol. 34, no. 5, pp. 380-391, 2014.

[20] A. Pereda, L. A. Vielva, A. Vegas, and A. Prieto, "Analyzing the stability of the FDTD technique by combining the von Neumann method with the Routh-Hurwitz criterion," IEEE Transactions on Microwave Theory and Techniques, vol. 49, no. 2, pp. 377-381, 2001.

[21] A. Grande, J. A. Pereda, O. Gonzalez, and N. Vegas, "On the equivalence of several FDTD formulations for modeling electromagnetic wave propagation in double-negative metamaterials," IEEE Antennas and Wireless Propagation Letters, vol. 6, pp. 324-327, 2007.

[22] J. A. Pereda, A. Vegas, and A. Prieto, "FDTD modeling of wave propagation in dispersive media by using the Mobius transformation technique," IEEE Transactions on Microwave Theory and Techniques, vol. 50, no. 7, pp. 1689-1695, 2002.

[23] Y. Q. Zhang and G. B. Ge, "An improved shift operator finite-difference time-domain method based on digital signal processing technique for general dispersive medium," Acta Physica Sinica, vol. 58, no. 12, pp. 8243-8248, 2009.
[24] O. Ramadan, "On the derivation of the numerical permittivity and stability of the CDS-FDTD implementation of high-order constitutive relations," IEEE Transactions on Antennas and Propagation, vol. 65, no. 3, pp. 1486-1489, 2017.

[25] Z. Lin and L. Thylen, "On the accuracy and stability of several widely used FDTD approaches for modeling Lorentz dielectrics," IEEE Transactions on Antennas and Propagation, vol. 57, no. 10, pp. 3378-3381, 2009.

[26] M. Okoniewaki, M. Mrozowski, and M. A. Stuchly, "Simple treatment of multi-term dispersion in FDTD," IEEE Microwave and Guided Wave Letters, vol. 7, no. 5, pp. 121-123, 1997. 


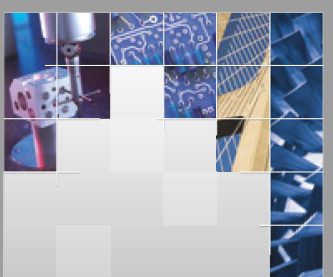

\section{Enfincering}
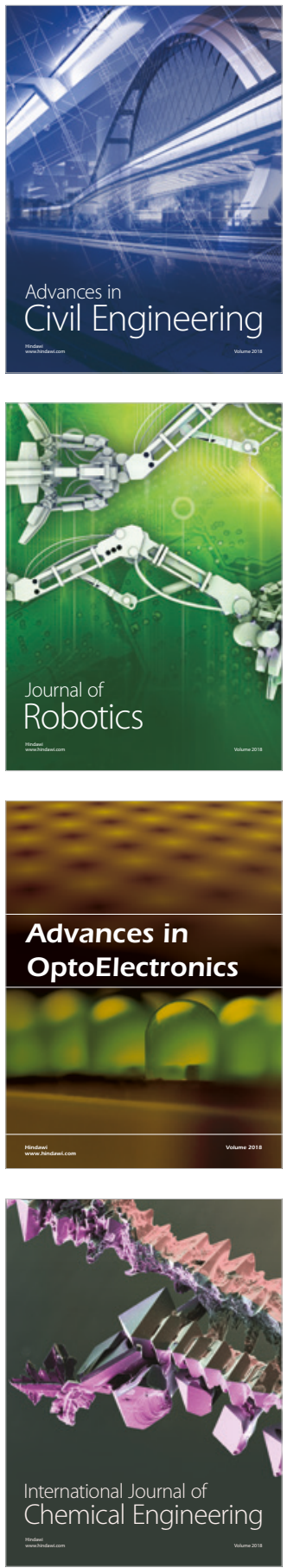

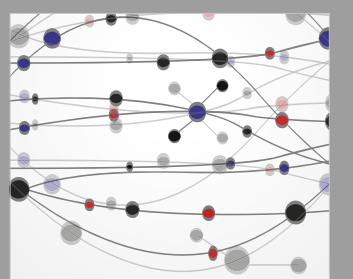

\section{Rotating \\ Machinery}

The Scientific World Journal

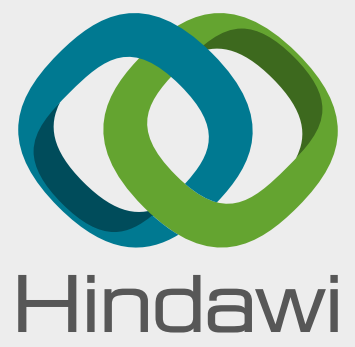

Submit your manuscripts at

www.hindawi.com
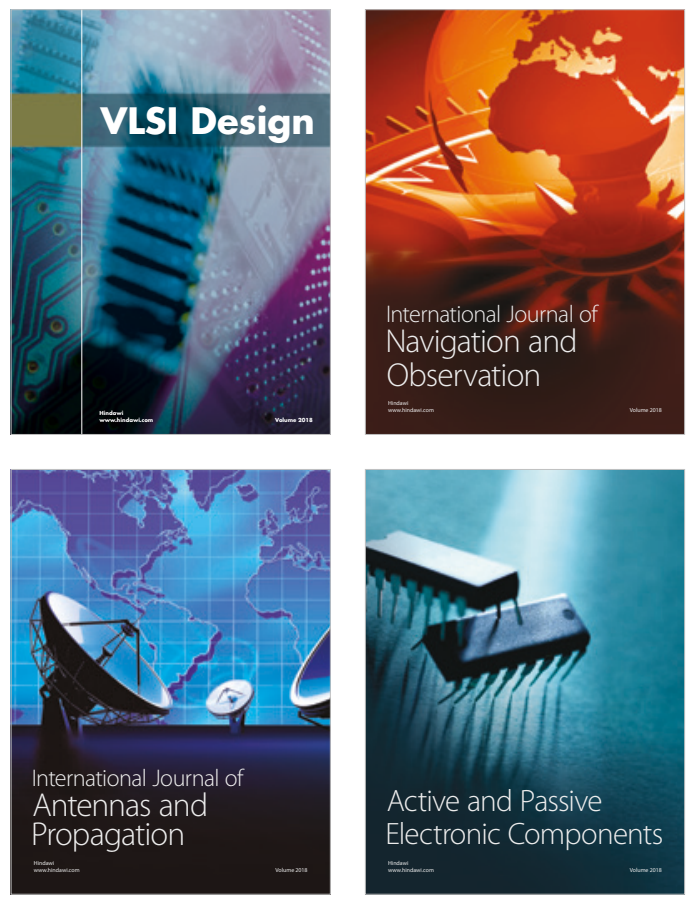
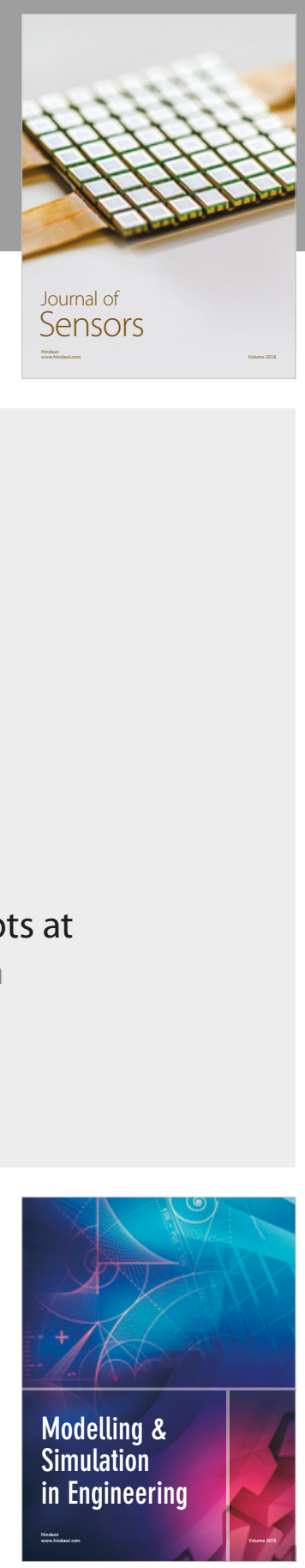

\section{Advances \\ Multimedia}
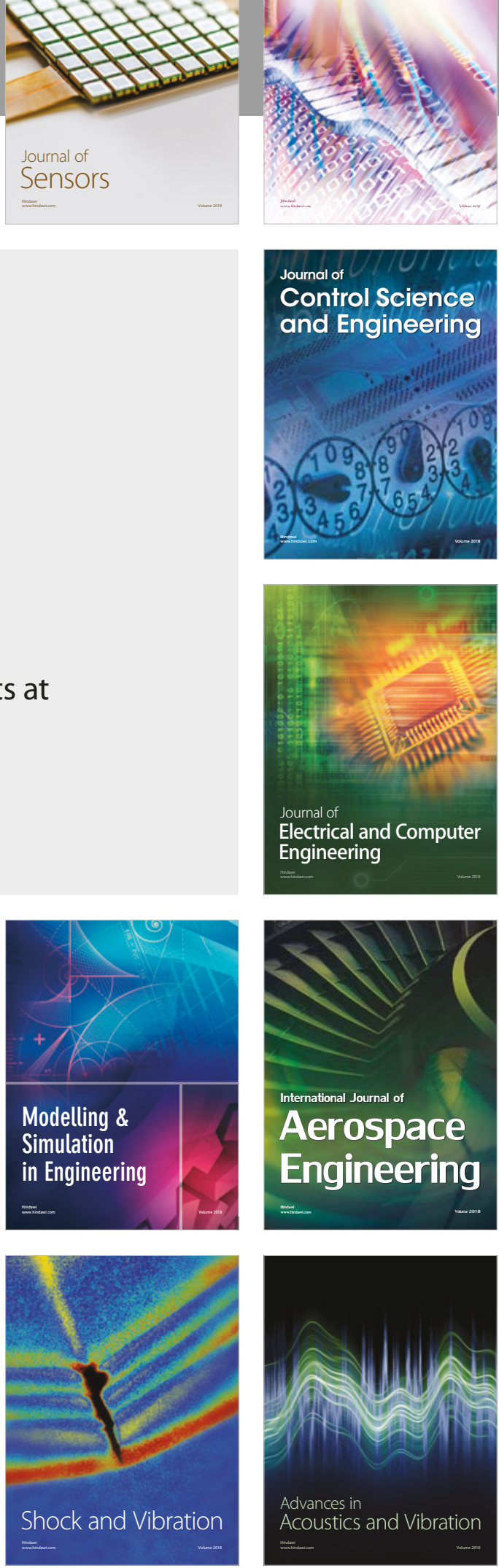